Please do not remove this page

RMIT

UNIVERSITY

\title{
An interference cancellation algorithm for fourier-based and wavelet-based OFDM systems
}

Abdullah, Khaizuran; Neville, Katrina; Hussain, Zahir

https://researchrepository.rmit.edu.au/esploro/outputs/9921864108001341/filesAndLinks?institution=61RMIT_INST\&index=null

Abdullah, K., Neville, K., \& Hussain, Z. (2008). An interference cancellation algorithm for fourier-based and wavelet-based OFDM systems. 2008 International Conference on Advanced Technologies for

Communications, 215-218. https://doi.org/10.1109/ATC.2008.4760557

Published Version: https://doi.org/10.1109/ATC.2008.4760557

Repository homepage: https://researchrepository.rmit.edu.au

(c) 2008 IEEE. Personal use of this material is permitted. However, permission to reprint/republish this material for advertising or promotional purposes or for creating new collective works for resale or redistribution to servers or lists, or to reuse any copyrighted component of this work in other works must be obtained from the IEEE.

Downloaded On 2023/04/27 00:24:19+1000 


\title{
An Interference Cancellation Algorithm for Fourier-Based and Wavelet-Based OFDM Systems
}

\author{
Khaizuran Abdullah, Katrina L. Neville, and Zahir M. Hussain, SMIEEE \\ School of Electrical \& Computer Engineering, RMIT University, Melbourne, Australia \\ khaizuran.abdullah@student.rmit.edu.au,katrina.neville@student.rmit.edu.au,zmhussain@ieee.org
}

\begin{abstract}
An Interference Cancellation Algorithm (ICA) for both Fourier- and wavelet-based OFDM is proposed. In this study we investigated both an ideal case as well as a non-ideal case of our system. In the ideal case we assume the reference signal is an unwanted sinusoidal signal (the input to our ICA algorithm). In the non-ideal case, we assume the received OFDM signal is contaminated with sinusoidal interference. Simulation results performed at a SNR of $5 \mathrm{~dB}$ resulted in an error measurement of 0.01812 for wavelet-OFDM and an error of approximately 0.03277 for Fourier-OFDM for the ideal case and for the nonideal case an error of 0.01817 was measured for wavelet-based OFDM and 0.03167 for Fourier-based OFDM. This indicates that the proposed ICA exhibits outstanding performance since the results obtained in both cases are almost the same. The waveletbased OFDM outperformed the Fourier-based OFDM in both cases.
\end{abstract}

-Keywords: Interference Cancellation Algorithm (ICA), Fourier-based OFDM, wavelet-based OFDM.

\section{INTRODUCTION}

An Orthogonal Frequency Division Multiplexing (OFDM) system is a multi-carrier system which utilises a parallel processing technique allowing the simultaneous transmission of data on many closely spaced, orthogonal sub-carriers. Conventional OFDM systems use the IFFT and FFT algorithms at the transmitter and recciver respectively to multiplex the signals together and decode the signal at the receiver. The system employs guard intervals or cyclic prefixes (CP) so that the delay spread of the channel becomes longer than the channel impulse response. The purpose of this is to minimize inter-symbol interference (ISI). however, the CP has the disadvantage of reducing the spectral containment of the channels [1],[3].

Other works published in the field have investigated the use of wavelet-based OFDM as a potential replacement for the Fourier-hased OFIMM and concluded that wavelet OFDM has many advantages over Fourier OFDM [1], [2], 13]. Comparisons between the performance of Fourier and wavelet OFDM have been discussed in [4]. [5]. Other works have also included interference signals in their work and have compared the performances of the two transforms taking this into account [2], [6].

The Interference Cancellation Algorithm (ICA) has been discussed by [8], this work included the use of windowing techniques to suppress thi interference. however, this work only examined Fourier-based OFDM. Our approach is to also include interference cancellation in wavelet-based OFDM.

In this paper we propose an ICA that can be implemented in both Fourier and wavelet-based OFDM systems.

\section{FOURIER-BASED OFDM}

A typical block diagram of an OFDM system is shown in Fig. 1, this shows the data $d_{k}$ first being processed by an M-ary QAM modulator to map the raw binary data to appropriate QAM symbols. These symbols are then input into the OFDM system. This involves taking $\mathrm{N}$ parallel streams of QAM symbols ( $\mathrm{N}$ being the number of sub-carriers used in the transmission of the data) and performing an IFFT operation on this parallel stream. The output in discrete time is as follows:

$$
x(n)=\frac{1}{\sqrt{N}} \sum_{k=0}^{N-1} X(k) \exp \left(j 2 \pi \frac{n}{N} k\right)
$$

where $x(n) \mid 0 \leq n \leq N-1$ is a sequence in the discrete time domain and $X(k) \mid 0 \leq k \leq N-1$ are complex numbers in the discrete frequency domain.

The cyclic prefix (CP) is lastly added before transmission occurs to minimize the inter-symbol interference (ISI). At the receiver, the process is reversed to obtain the decoded data. The $\mathrm{CP}$ is removed to obtain the data in the discrete time domain. The data is then processed by the ICA block to eliminate any unwanted sinusoidal interference before an FFT is performed on the data. The output of the FFT in the frequency domain is as follows:

$$
X(k)=\sum_{n=0}^{N-1} x(n) \exp \left(-j 2 \pi \frac{n}{N} k\right)
$$

\section{WAVELET-BASED OFDM}

The wavelet transform blocks comprise of an inverse discrete wavelet transform (IDWT) at the transmitter and a discrete wavelet transform (DWT) at the receiver, these blocks in effect replace the IFFT and FFT blocks discussed in Section II. Due to the overlapping nature of wavelets, the waveletbased OFDM does not need a cyclic prefix to deal with the delay spreads of the channel. As a result, it has a higher spectral containment than in Fourier OFDM [1].

In wavelet OFDM the data is, in effect, processed the same as discussed in Section II, however, the cyclic prefix block is 


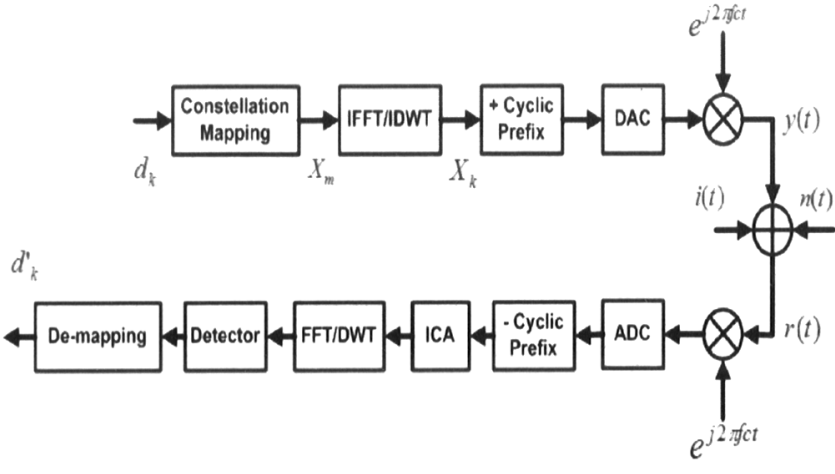

Fig. 1. A Typical model of an OFDM transceiver with an insertion on the proposed ICA

removed. The output of the inverse discrete wavelet transform (IDWT) can be represented as [4]:

$$
s(k)=m \sum_{m=0}^{\infty} \sum_{n=0}^{\infty} S_{m}^{n} 2^{\frac{m}{2}} \phi\left(2_{k}^{m}-n\right)
$$

where $S_{m}^{n}$ are the wavelet coefficients and $\phi\left(2_{k}^{m}-n\right)$ is the wavelet function with a compression factor of $m$ times and a shift factor of $\mathrm{n}$ times for each subcarrier (numberk, $0 \leq k \leq$ $N-1)$.

The wavelet coefficients are the representation of the signal in scale and position or time. The scale is related to the frequency of the signal; low scale represents a compressed wavelet where the signal is rapidly changing (signal is of a high frequency) and high scale represents a stretched wavelet where the signal is slowly changing (signal is of a low frequency). Thus, $X(k)$ can be represented as $S_{m}^{n}$ before it is processed by the IDWT. The output of the discrete wavelet transform (DWT) at the receiver is:

$$
S_{m}^{n}=\sum_{k=0}^{N-1} s(k) 2^{\frac{m}{2}} \phi\left(2_{k}^{m}-n\right)
$$

$S_{m}^{n}$ can be decoded to $X(k)$ before the recovered data is processed by the QAM demodulator.

\section{SySTEM MODEL WiTH ICA}

A typical OFDM transmitter was discussed in Sections II and III. In this paper, we also include an ICA component before the signal is processed by the FFT/DWT block. A discrete-time domain baseband signal at the front-end of the receiver can be modelled as follows:

$$
y(n)=r(n)+g(n)+i(n)
$$

where $y(n)$ is the received OFDM signal in the discrete time domain and consists of the OFDM transmitted signal $r(n)$ corrupted with additive white Gaussian noise $g(n)$, and an unwanted sinusoidal signal $i(n)$. We assume that $i(n)$ is the dominant part that corrupts the OFDM signal. The baseband signal $i(n)$ component can be modelled as follows:

$$
i(n)=A \sin \left(2 \pi f \frac{n}{N}+\phi\right)
$$

where $A, f$ and $\phi$ are the amplitude, frequency and phase of the signal respectively. The discrete-time signal $i(n)$ has $n$ samples with $N$ sub-carriers. The model in equation 6 is relatively simple and requires little complex mathematics to extract and minimize the interference signal. Previously, a very similar model was used in [7] and [9]. The work in [7], however, included multiple parameters indicated by the subscript $i$ in $A_{i}, f_{i}$ and $\phi_{i}$ and there was no indication that this model was used for an OFDM system. On the other hand, in [9] the similar model was used without parameter $\phi$ for a direct-sequence spread spectrum system.

\section{INTERFERENCE CANCELLATION ALGORITHM (ICA) IN FOURIER-BASED AND WAVELET-BASED OFDM}

When the system receives the signal $y(n)$, all three components in (5) are serial signals. If Fourier-based OFDM is implemented then the signal will also comprise of a Cyclic Prefix (CP) which will inevitably need to be removed before further processing [1], [3], [4].

At this point the serial data is passed into the ICA block where the main task is to isolate the unknown signal component $i(n)$ corrupting the data stream. A flow-chart of the proposed ICA algorithm used in this work is detailed in Fig. 2.

Before the ICA can perform the task of detecting the unknown signal component, all the variables such as the length of the test signal $k$, the error signal $e$, the error squared $\bar{e}^{2}$ and the mean-squared error $E$ have to be initialized. Next the ICA will call the testing signals consisting of the estimating parameters $\widehat{A}, \widehat{f}$ and $\widehat{\phi}$ as follows:

$$
\widehat{i}_{\text {test }}(n)=\left[\widehat{A}_{\text {test }}\right] \sin \left(2 \pi\left[\widehat{f}_{\text {test }}\right] \frac{n}{N}+\left[\widehat{\phi}_{\text {test }}\right]\right)
$$

where $\widehat{A}_{\text {test }}=\left[\widehat{A}_{k=1: K}\right], \widehat{f}_{\text {test }}=\left[\widehat{f}_{k=1: K}\right]$ and $\widehat{\phi}_{\text {test }}=$ $\left[\widehat{\phi}_{k=1: K}\right] . K$ is also chosen to have an odd number greater or equal to 3 . For a better understanding, we can further describe the process by expanding (7) as follows:

$\widehat{i}_{\text {test }}(n)=\left[\widehat{A}_{\text {est }} \in \widehat{A}_{1}, \widehat{A}_{2}, \ldots, \widehat{A}_{K}\right] \sin \left(2 \pi\left[\widehat{f}_{\text {test }}\right] \frac{n}{N}+\left[\widehat{\phi}_{\text {test }}\right]\right)$

where $\widehat{A}_{\text {est }}$ is the desired amplitude estimated by $\widehat{A}_{\text {test }}$. After the processing and detection in (8), the ICA will continue to detect the frequency. The equation will then be updated to:

$$
\widehat{i}_{\text {test }}(n)=\left[\widehat{A}_{\text {est }}\right] \sin \left(2 \pi\left[\widehat{f}_{\text {est }} \in \widehat{f}_{1}, \widehat{f}_{2}, \ldots, \widehat{f}_{K}\right] \frac{n}{N}+\left[\widehat{\phi}_{\text {test }}\right]\right)
$$

where $\widehat{f}_{\text {est }}$ is the desired frequency estimated by $\widehat{f}_{\text {test }}$.

Next the ICA will update the task to get the estimations $\hat{A}_{\text {est }}$ and $\widehat{f}_{e s t}$, and it will proceed to estimate the last parameter, $\widehat{\phi}_{\text {est }}$. We will then have the following equation:

$$
\widehat{i}_{\text {test }}(n)=\left[\widehat{A}_{\text {est }}\right] \sin \left(2 \pi\left[\widehat{f}_{\text {est }}\right] \frac{n}{N}+\left[\widehat{\phi}_{\text {est }} \in \widehat{\phi}_{1}, \widehat{\phi}_{2}, \ldots, \widehat{\phi}_{K}\right]\right)
$$




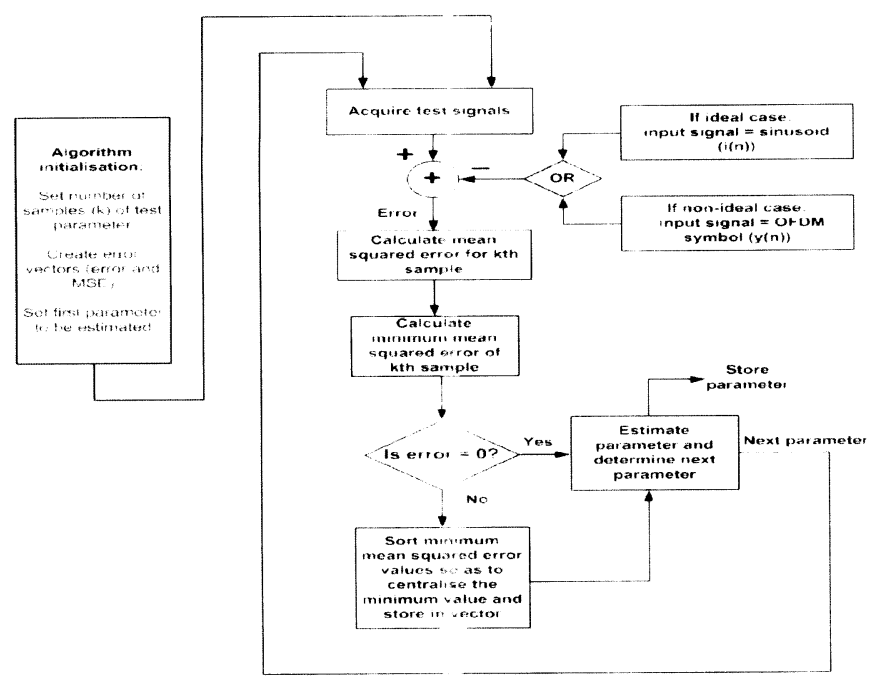

Fig. 2. The proposed Interference Cancellation Algorithm. (ICA)

The ICA will finalize the estimated interference signal having all estimated parameters as follows

$$
\widehat{i}_{\text {test }}(n)=\left[\widehat{A}_{\epsilon s t}\right] \sin \left(2 \pi\left[\widehat{f}_{\text {est }}\right] \frac{n}{N}+\left[\hat{\phi}_{\text {est }}\right]\right)
$$

Sections V-A and V-B will describe how the ICA takes different steps to obtain (11) for both ideal and non-ideal cases.

\section{A. Ideal Case}

The ideal case occurs when the interference model $i(n)$ in (6) is used to subtract the testing signals. The purpose of this is to obtain a reference measurement to evaluate the performance of the ICA in a practical situation. The result of the subtraction of (6) and (8) will be:

$$
\widehat{e}(n)=i(n)-\hat{i}_{\text {test }}(n)
$$

or

$$
\begin{array}{r}
\hat{e}(n)=\left[A-\widehat{A}_{\text {test }} \sin \left(2 \pi\left[\widehat{f}_{\text {test }}\right] \frac{n}{N}+\left[\hat{\phi}_{\text {test }}\right]\right)\right. \\
\widehat{e}(n)=\left[A-\widehat{A}_{\text {est }} \in \lambda\right] \sin \left(2 \pi\left[\hat{f}_{\text {test }}\right] \frac{n}{N}+\left[\widehat{\phi}_{\text {test }}\right]\right)
\end{array}
$$

where $\chi=\left[\left\langle A-\hat{A}_{1}\right\rangle,\left\langle A-\hat{A}_{2}\right\rangle, \ldots,\left\langle A-\hat{A}_{K}\right\rangle\right]$. Taking the error square and mean of (14) yiclds:

$$
E\left[\widehat{e}^{2}(n)\right]= \begin{cases}0, & \text { if } A=\widehat{A} \ldots \\ \varepsilon, & \text { if } A \neq \widehat{A} \ldots\end{cases}
$$

where $\varepsilon$ is the residue of the subtraction in (14).

Referring to Fig. 2 it can be seen that the ICA is a mostly iterative algorithm that continues to loop while $E=\varepsilon$, as soon as the case occurs where $E=0$ the ICA will stop the loop and store the result of the amplitude parameter estimation $\widehat{A}_{e s t}$. it will then look for the next desired parameter to be estimated and this, in turn, will re-initialise the loop. This same process will be repeated to estimate all the desired parameters in the signal.

At the end of the algorithm $\widehat{A}_{\text {est }}, \hat{f}_{\text {est }}$ and $\widehat{\phi}_{\text {est }}$ will be detected and stored. The results will then be passed into the demodulation block and a final error is calculated.

\section{B. Non-Ideal Case}

For the non-ideal case, we consider $y(n)$ (a received OFDM signal with cyclic prefix removed) as the input to the ICA. The reason it is called non-ideal is because it uses the received signal as the input to the ICA instead of $i(n)$. This is illustrated in Fig. 2. In this non-idea case, the equations from (7) to (11) are repeated as previously described, however, (12) is required to be changed to $\widehat{e}(n)=y(n)-\widehat{i}_{\text {test }}(n)$. Basically from now the same mathematical equations discussed in Section V-A can be used. $\widehat{e}(n)$ can be rewritten as:

$$
\hat{e}(n)=\left[\zeta+i(n)-\widehat{A}_{\text {test }}\right] \sin \left(2 \pi\left[\widehat{f}_{\text {test }}\right] \frac{n}{N}+\left[\widehat{\phi}_{\text {test }}\right]\right)
$$

Since we are interested in cancelling out $i(n),(16)$ can be expanded to:

$$
\widehat{e}(n)=\left[\zeta+\left\langle A-\widehat{A}_{\text {test }}\right\rangle \in \chi\right] \sin \left(2 \pi\left[\widehat{f}_{\text {test }}\right] \frac{n}{N}+\left[\widehat{\phi}_{\text {test }}\right]\right)
$$

where, $\chi=\left[\left\langle A-\widehat{A}_{1}\right\rangle,\left\langle A-\widehat{A}_{2}\right\rangle, \ldots,\left\langle A-\widehat{A}_{K}\right\rangle\right]$. Then taking the error square and mean of (17) yields:

$$
E\left[\widehat{c}^{2}(n)\right]=\left\{\bar{\sigma}, \widehat{A}_{\text {est }} \in\left[\left\langle A-\widehat{A}_{1}\right\rangle,\left\langle A-\widehat{A}_{2}\right\rangle, \ldots,\left\langle A-\widehat{A}_{K}\right\rangle\right]\right.
$$

where $\bar{\sigma}=\min \left(E\left[\widehat{e}^{2}(n)\right]\right)$ and $\bar{\sigma}$ are complex.

For simplicity, the ICA will sort the elements of $\bar{\sigma}$ and arrange its minimum value in the mid-index location. It can be assumed that the actual parameters are approximately in the mid-index location of the testing parameter range since $K$ is assumed to be odd, the ICA will determine the estimated parameter based on the index corresponding to the minimum value in the mid-index location. Again as discussed in Section V-A the ICA will determine $\widehat{A}_{e s t}, \hat{f}_{\text {est }}$ and $\widehat{\phi}_{\text {est }}$ and store them before passing them to the FFT or DWT block.

\section{PERFormance of AN OFDM System: SinUsoidal INTERFERENCE}

MATLAB was used to perform the simulation. Performance of an OFDM system in the presence of an unwanted sinusoidal signal as compared to a system without it is shown in Fig. 3. For the case of $\mathrm{SNR}=5 \mathrm{~dB}$ the error was 0.04578 as compared to 0.01853 without interference. The difference of about 2.7 percent error was due to the fact that the system performance was affected by the sinusoidal interference.

\section{Perrormance of ICA: Ideal CaSh}

Figure 4 shows the performance of an ideal case. Waveletbased OFDM was shown to outperform Fourier-based OFDM. This again can be seen in the case of SNR $=5 \mathrm{~dB}$ where an error of 0.01812 was obtained for wavelet-OFDM and 0.03277 for the Fourier-OFDM. As SNR increases, the wavelet-OFDM continues to outperform Fourier-based OFDM. This was true since the input for the subtraction from the acquired test signals was the sinusoidal interference itself. Because of this, the minimum mean square error (MMSE) would obtain zero. In addition, the property of wavelet transform is it does not required any $\mathrm{CP}$. The $\mathrm{CP}$ has the disadvantage of reducing the spectral containment. 


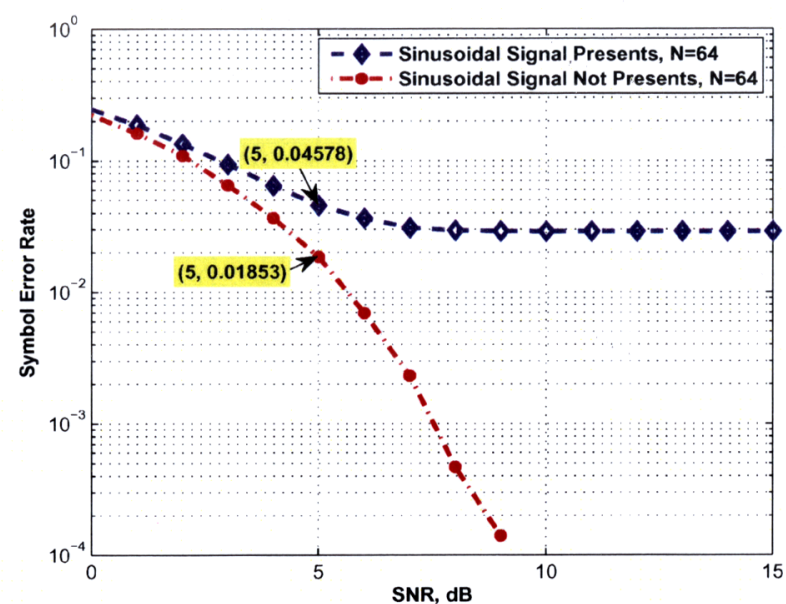

Fig. 3. Performance of an OFDM system with and without interference.

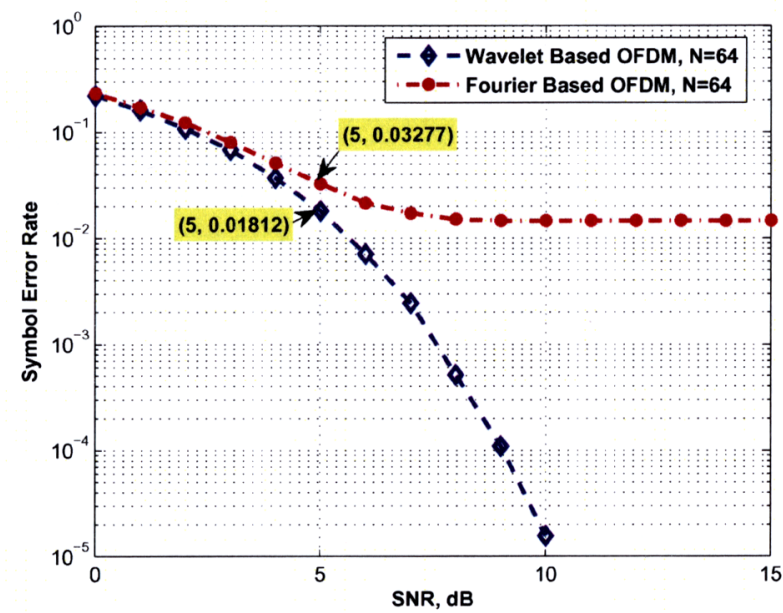

Fig. 4. Performance of Fourier- and Wavelet-Based OFDM: the Ideal Case.

\section{PERFormance of ICA: Non-IDEAL CASE}

For the non-ideal case, we assumed that all testing parameters are positive. This is important since our interference model is a sine wave which is an odd function, as opposed to a cosine wave signal. We also assumed that the number of samples were odd. This was because the ICA performed the tasks of sorting and centralising the minimum value after determining MMSE. Figure 5 shows the performance of the non-ideal case.

For this non-ideal case, the wavelet-based OFDM has also outperformed Fourier-based OFDM. At SNR $=5 \mathrm{~dB}$, an error of 0.01817 was obtained as compared to 0.03167 . The difference of about 1.35 percent was due to the fact that wavelet transform has the property as described in the previous section. The system also shows that the ideal and non-ideal

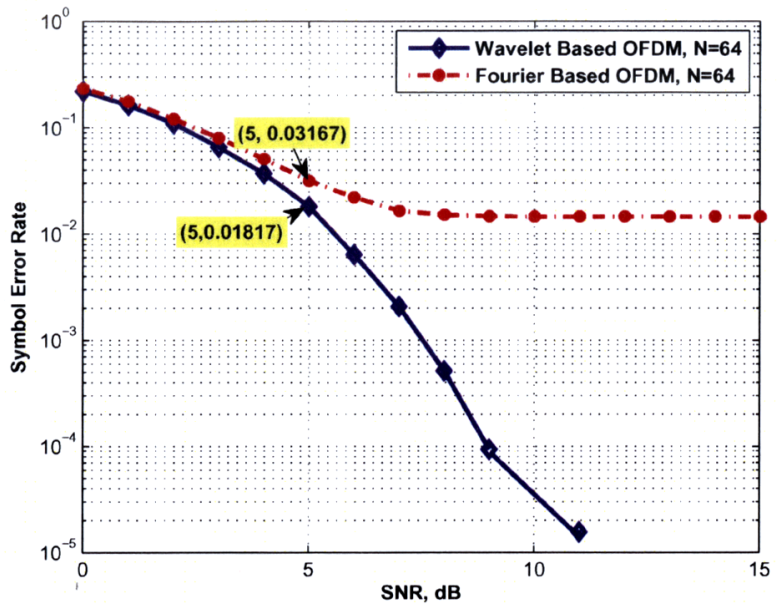

Fig. 5. Performance of Fourier- and wavelet-based OFDM: a nonideal Case.

cases have approximately the same curve. This means that the proposed ICA algorithm has a good performance.

\section{CONCLUSION}

We proposed an interference cancellation algorithm (ICA) that has shown outstanding performance for both ideal and non-ideal system cases where it reached almost the same error. A sinusoidal interfering signal was considered. In addition, the wavelet-based OFDM outperformed Fourier-based OFDM in both cases. This paper considered an estimation of a single type of interference but it is expected that in future work the model described in this work can be expanded to consider multiple interferences as mentioned in work by [2]. It is also good if this work can be extended to include other interference cancellation or suppression algorithms in future work.

\section{REFERENCES}

[1] S. D. Sandberg, and M. A. Tzannes, "Overlapped Discrete Multitone Modulation for High Speed Copper Wire Communications", IEEE Journal on Selected Areas in Communications, vol. 13, no. 9, pp. 1571-1585, 1995.

[2] A. N. Akansu, and L. Xueming, "A Comparative Performance Evaluation of DMT (OFDM) and DWMT (DSBMT) Based DSL Communications Systems for Single and Multitone Interference", Proceedings of the IEEE International Conference on Acoustics, Speech and Signal Processing, vol. 6, pp. 3269 - 3272, May 1998.

[3] N. Ahmed, "Joint Detection Strategies for Orthogonal Frequency Division Multiplexing", Dissertation for Master of Science, Rice University, Houston, Texas. pp. 1-51, April 2000.

[4] K. Abdullah. and Z. M. Hussain, "Performance of Fourier-Based and Wavelet-Based OFDM for DVB-T Systems", IEEE Australasian Telecommunication Networks and Applications Conference, Dec 2007.

[5] S. R. Baig, F. U. Rehman, and M. J. Mughal, "Performance Comparison of DFT, Discrete Wavelet Packet and Wavelet Transforms in an OFDM Transceiver for Multipath Fading Channel", 9th IEEE International Multitopic Conference,, pp. 1-6, Dec 2005.

[6] Y. Wang, and X. P. Zhang, "Filter bank based interference suppression for fading channels", IEEE Canadian Conference on Electrical and Computer Engineering, vol. 2, pp. 961- 964 May 2003.

[7] L. Xu, and Y. Yan, "Wavelet-based Removal of Sinusoidal Interference from a Signal", IOP Electronic Journals: Measurement Science and Technology, vol. 15, no. 9, pp. 1779-1786, 2004. 
[8] S. Kapoor, and S. Nedic, "Interference Suppressions in DMT Receiver Using Windowing", IEEE International Conference on Communications, vol. 2, pp. 778-782, June 2000 .

[9] J. G. Proakis, M. Salehi, and G. Bauch, Contemporary Communication Systems Using MATLAB Second ed. Brooks Cole. 2004. 